\title{
Successful treatment of isolated bile leakage after hepatectomy combination therapy with percutaneous transhepatic portal embolization and bile duct ablation with ethanol: a case report
}

\author{
Norio Kubo ${ }^{1}$, Norifumi Harimoto ${ }^{1 *}$, Kei Shibuya ${ }^{2}$, Norihiro Ishii ${ }^{1}$, Mariko Tsukagoshi ${ }^{1}$, Takamichi Igarashi ${ }^{1}$,
} Akira Watanabe ${ }^{1}$, Kenichiro Araki ${ }^{1}$, Masaya Miyazaki ${ }^{2}$, Hiroyuki Kuwano ${ }^{3}$ and Ken Shirabe ${ }^{1}$

\begin{abstract}
Background: Bile leakage after hepatectomy still causes relatively serious problems, and some types of bile leakage are intractable.

Case presentation: We report a case of postoperative isolated bile duct leakage managed successfully by combination therapy of percutaneous transhepatic portal vein embolization (PTPE) and bile duct ablation with ethanol. A 61-year-old man diagnosed with hepatocellular carcinoma underwent partial hepatectomy. On postoperative day 1, bile leakage was detected at the drainage tube. Simple drainage treatment did not improve the situation. He was diagnosed with isolated bile leakage based on fistulogram from the drainage tube that showed the bile duct at segments V and VIII but not the common bile duct. A volume of drainage fluid of $200 \mathrm{~mL} /$ day was observed. Combination therapy with PTPE and bile duct ablation with ethanol was planned. After the percutaneous transhepatic cholangiography, the drainage tube was inserted into the bile duct, and PTPE was performed to segments V and VIII. The amount of drainage fluid decreased, and bile duct ablation with ethanol was performed to the isolated bile duct. No complication was found following combination therapy.
\end{abstract}

Conclusion: In this case, we successfully treated a patient with isolated bile leakage by combination therapy with PTPE and bile duct ablation.

Keywords: Bile leakage, Divided bile duct, Isolated bile duct, Segmental bile leakage, Percutaneous transhepatic portal embolization, Bile duct ablation

\section{Background}

Recently, it has been possible to perform hepatectomy safely because of improvement of surgical techniques and perioperative management $[1,2]$. Recent studies reported that perioperative mortality rates were 2.6 to $3 \%$ after major hepatectomy $[3,4]$. However, bile leakage has still a relatively high incidence in the range of $4.8-8.7 \%[1,2,5]$.

\footnotetext{
* Correspondence: nharimotoh1@gunma-u.ac.jp

'Department of Hepatobiliary and Pancreatic Surgery, Gunma University,

Graduate School of Medicine, 3-39-22 Showa-machi, Maebashi, Gunma

371-8511, Japan

Full list of author information is available at the end of the article
}

Bile leakage may lead to intraperitoneal septic complications, liver failure, and ultimately death. The risk factors for bile leakage have been already described previously [6]. Reported factors that were correlated with the occurrence of bile leakage were male sex, advanced age, tumor size, repeated hepatectomy, preoperative chemoembolization, large liver cut surface, exposure of Glisson's sheath, duration of vascular occlusion, prolonged operation time, and red cell transfusion [6-9]. Bile leakage after hepatectomy was defined according to the international study group of liver surgery definition, i.e., drainage of fluid with a bilirubin level of three times greater than the serum level after 
postoperative day (POD) 3 or the need for interventions as the result of bile collections or biliary peritonitis [10]. Most bile leakages are treated with simple drainage. However, some types of bile leakage require interventions such as endoscopic bile drainage or percutaneous abdominal drainage. Furthermore, intractable bile leakage as represented by isolated bile leakage was not improved by drainage alone.

In this report, we present a patient with isolated bile duct leakage after hepatectomy who was successfully treated by combination therapy with percutaneous transhepatic portal embolization and bile duct ablation with ethanol without laparotomy.

\section{Case presentation}

A 61-year-old man underwent resection of a part of his tongue due to tongue cancer and was admitted to our hospital for hepatocellular carcinoma with about $5 \mathrm{~cm}$ diameter of tumor at the liver segment IV. Computed tomography (CT) showed that the tumor was enhanced during the arterial phase and washed out during the portal phase, and the tumor pressed the right anterior branch to the main branch of Glisson and the middle hepatic vein; hence, the diagnosis of hepatocellular carcinoma was made (Fig. 1). The indocyanine green retention rate at 15 min was $21.8 \%$. Child-Pugh score was A. The patient tested negative for hepatitis B surface antigen and hepatitis $\mathrm{C}$ virus antibody. He had a history of excessive consumption of alcohol, and alcoholic liver damage was considered as a possibility. Partial hepatectomy with segments IV $+\mathrm{V}$ and cholecystectomy with cystic duct-tube drainage were performed. Intraoperative findings indicated that the anterior branch of bile duct was exposed at the resected area, and some small bile ducts were ligated. On POD 1 following hepatectomy, bile leakage developed from the drain placed in the foramen of Winslow. CT showed fluid collection in the

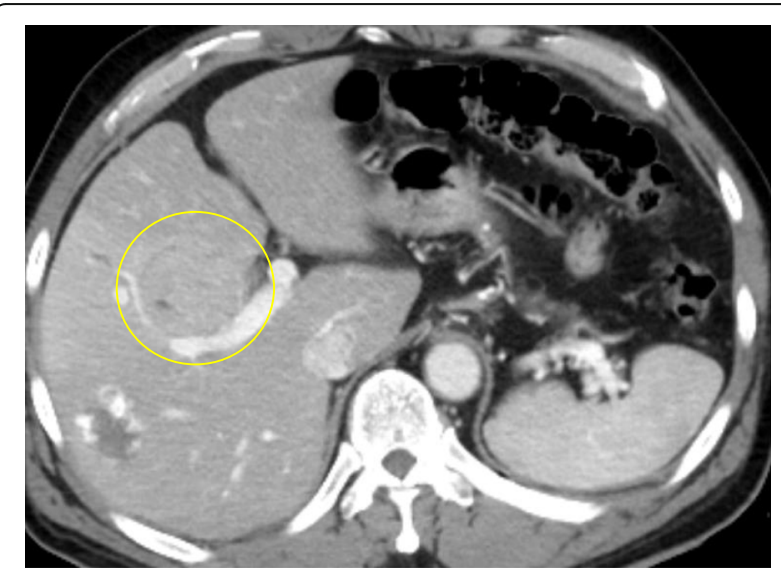

Fig. 1 Preoperative abdominal computed tomography showed a $50 \times 48 \mathrm{~mm}$ hepatic mass that pressed the anterior Glisson cavity between the liver and fistula to drain. Cholangiography via the endoscopic bile duct enhancement showed no communication between the common bile duct and abdominal cavity. Drip infusion cholangiography (DIC)-CT revealed the bile duct of the peripheral side. We maintained simple drainage to reduce the cavity. On POD 19, this patient had fever and CT revealed that the fluid collection has increased (Fig. 2a). Percutaneous drainage to the cavity near the liver cut surface was performed. Cholangiography via the c-tube did not show the anterior branch of the bile duct. Fistulogram from the drainage tube at the abdominal cavity showed the bile duct at segments V and VIII (Fig. 2b). We diagnosed the bile leakage from the isolated bile duct of segments $\mathrm{V}$ and VIII. Further management was needed to control the persistent biliary leak of 200-250 mL/day. Liver function was evaluated again. The indocyanine green retention rate at $15 \mathrm{~min}$ was $27.7 \%$. LHL15 was 0.575 . Liver volume of segments V and VIII was $260 \mathrm{~mL}$, and remnant liver volume was $1272 \mathrm{~mL}$, which were calculated using the 3D image analysis system (SYNAPSE VINCENT; Fuji Photo Film Co., Ltd.). Functional remnant liver volume (FRLV) was calculated based on the liver volume using gadolinium-ethoxybenzyl -diethylenetriamine pentaacetic acid-enhanced MRI for 20 min [11]. FRLV after liver resection of segments V and VIII was $2176 \mathrm{~mL}$. Even if the function becomes extinct by PTPE to segment V and VIII, remnant liver volume was sufficient. We considered that bile leakage could not be cured with either PTPE or bile duct ablation alone, because of the large volume of bile leakage more than $200 \mathrm{~mL}$ per day. The therapeutic strategy involved combination therapy of PTPE and bile duct ablation. First, percutaneous transhepatic cholangiography drainage (PTCD) tube was inserted to the bile duct of segment V. PTPE with coil embolization was performed to the part of the portal vein of segments $\mathrm{V}$ and VIII by puncturing the part of the portal vein of segment V. The liver volume of segments V and VIII was decreased from 260 to $123 \mathrm{~mL}$ after PTPE. After the PTPE, bile leakage decreased to about $50 \mathrm{~mL} /$ day. We confirmed that cholangiography via the PTCD tube showed the bile duct at segments V and VIII. For bile duct ablation, $1.2 \mathrm{~mL}$ pure ethanol was injected from the PTCD tube. Over $1.2 \mathrm{~mL}$ of ethanol leaked into the abdominal cavity. After ethanol injection, the PTCD tubes were clamped for $5 \mathrm{~min}$. Then, another bile duct ablation with $2.0 \mathrm{~mL}$ of pure ethanol was performed 1 week after of the first procedure. After bile duct ablation, bile leakage has decreased from 50 to $10 \mathrm{~mL} /$ day gradually. The patient left the hospital, and he was rehospitalized and reinjected with $2.0 \mathrm{~mL}$ of pure ethanol for three times. The drainage tube and bile duct tube were removed on POD 139 (Fig. 3). The clinical course is 

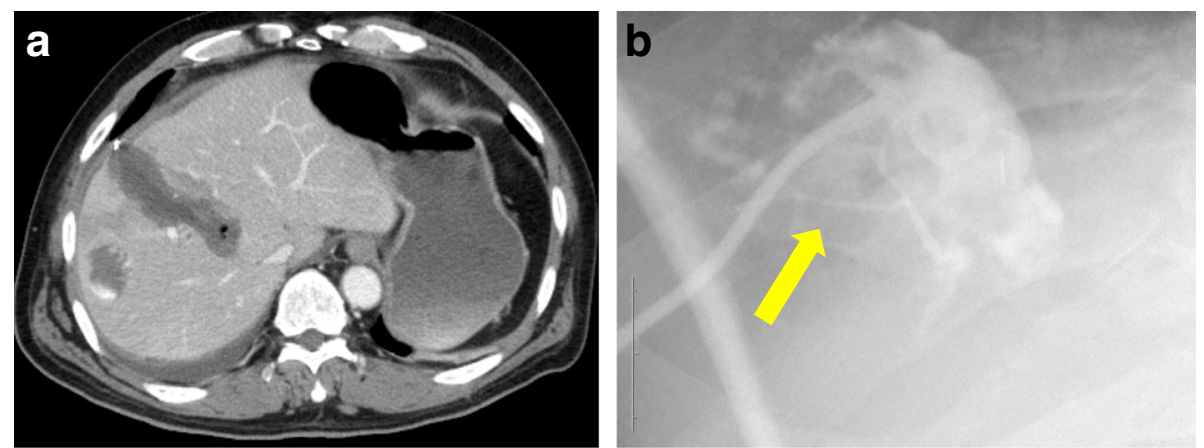

Fig. 2 On postoperative day 19, abdominal computed tomography showed the fluid th the liver cut surface (a). Cholangiography via the c-tube did not detect the anterior branch of the bile duct (arrows) and does not communicate with the abdominal cavity (b)

summarized in figure. Complications with combination treatment of PTPE and bile duct ablation were not noted.

\section{Discussion}

Bile leakage is categorized into four types by Nagano et al.: type 1 is minor leakage from the cut surface, type 2 is leakage caused by insufficient closure of the bile duct stump, type 3 is leakage from the injured bile duct wall at exposed bile duct or hilar bile duct, and type 4 is leakage from the distal orifice of the isolated bile duct $[5,9]$. The present case was classified as type 4 .

Therapeutic methods for isolated bile leakage can be divided into surgical and non-surgical methods. Surgical treatment was adapted to intractable bile leakage which was not treated by non-surgical management. Fukuhisa et al. reported the successful treatment of a case by surgical treatment of the isolated bile leakage from the Spiegel lobe after right hemihepatectomy [12]. They suggested that surgical treatment with liver resection for postoperative isolated bile leakage was both a quick and reliable procedure for patients with acceptable liver function and anatomical subject [12]. Fistulojejunostomy was one of the therapeutic methods reported [13], but a detailed report is unavailable. In this patient, isolated bile leakage was diagnosed using DIC-CT and cholangiography, with the drainage tube or endoscopic method. It was considered that it was not cured by drainage alone. Anterior resection after partial hepatectomy was not technically easy, and the patient was not hoped. Fistulojejunostomy to the leaked bile duct was difficult because the sutured space was transformed into a slit shape. Furthermore, this operation was thought to be difficult for a patient with hepatocellular carcinoma and cirrhosis

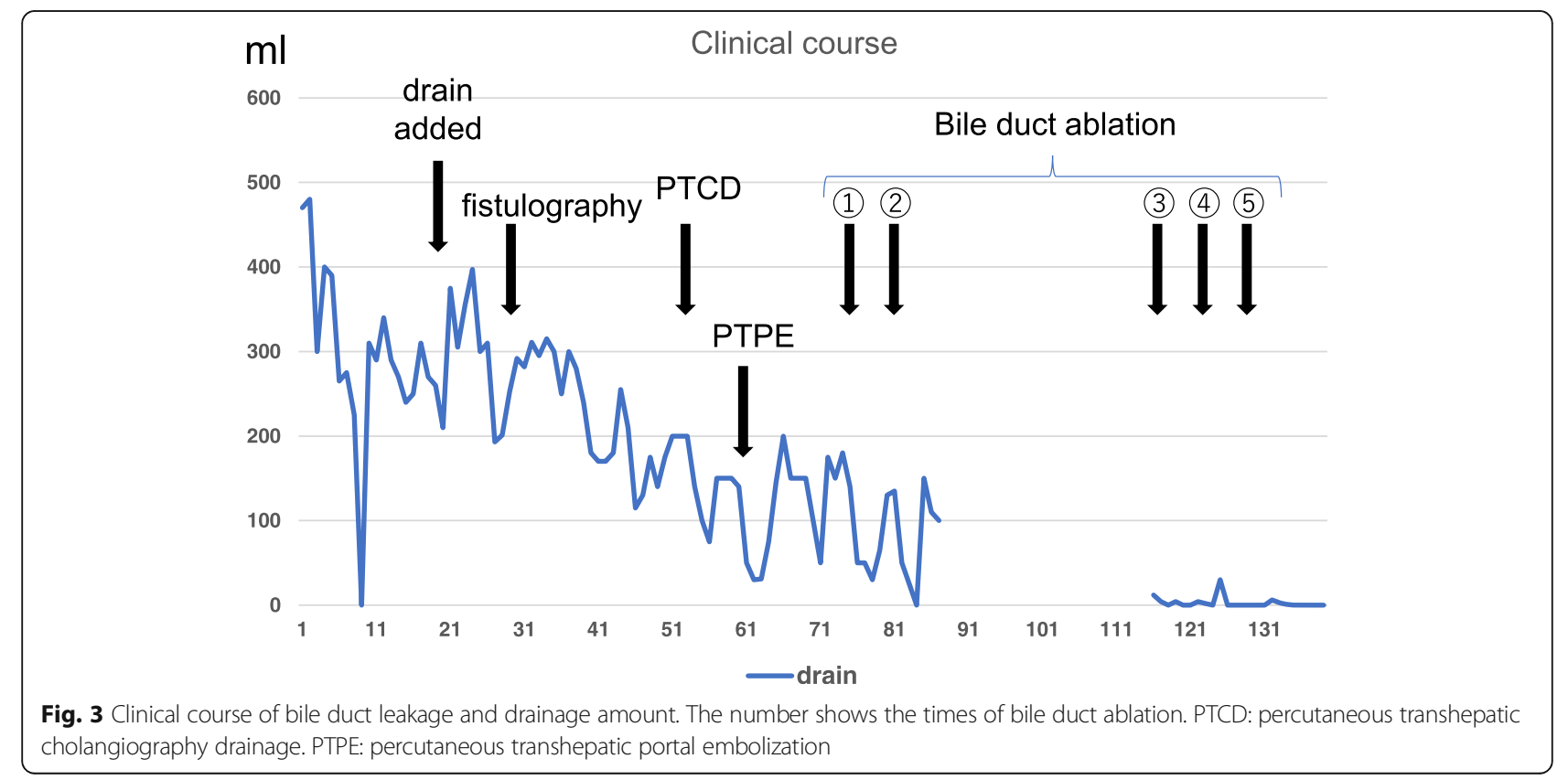


because treatments such as transcatheter arterial chemoembolization or radiofrequency ablation for the recurrence of HCC are contraindication because treatment with transarterial chemoembolization and radiofrequent ablation becomes high risk of the liver abscess [14].

Non-surgical treatment for isolated bile leakage requires interventions such as PTPE, embolization with fibrin glue, and bile duct ablation. PTPE was one of the therapeutic methods for intractable bile leakage, and the use of PTPE decreased bile leakage quantity due to the embolus of the responsible bile duct domain. Sadakari et al. reported a successfully treated case of bile leakage from isolated bile duct of the posterior segment by PTPE which induces atrophy of hepatocytes [15]. Tanaka et al. reported that the isolated bile leakage was cured by sealing the duct with a mixture of fibrin glue and iodized oil [16]. However, this treatment did not cause infection, but a few bile leakages were noted. Bile duct ablation therapy was reported for the first time by Kyokane et al. [17].

When segments V and VIII were resected, the FRLV was $2176 \mathrm{~mL}$. Itoh et al. showed the cutoff value of liver-related morbidity was $731 \mathrm{~mL}$ [11]. Therefore, it was thought that it has a low risk of liver failure even if segments V and VIII of the liver became dysfunctional. Previous reports have shown successful treatment of bile leakage with bile duct ablation when the amount of bile leakage was $50-150 \mathrm{~mL}$ [18-20]. PTPE was performed when the amount of bile leakage was $40-50 \mathrm{~mL}[15,21]$. In this case, the amount of bile leakage from segments $\mathrm{V}$ and VIII was more than $200 \mathrm{~mL} /$ day. It was considered that PTPE or bile duct ablation therapy alone could not stop the bile leakage. PTPE reduces hepatocellular function and decreases bile production. Kyokane reported that, in an animal study, selective intrahepatic biliary ethanol injection destroyed the biliary epithelium, permeated the parenchyma, induced hepatocyte degeneration, and resulted in compensatory hypertrophy of the non-injective hepatic lobe [22]. We thought that it was appropriate to perform bile duct ablation after decreasing bile production with PTPE. We performed the combination therapy with PTPE and bile duct ablation with pure ethanol. First, a PTCD tube was inserted into the leaking bile duct for a direct route, applying pure ethanol, which had originally been planned after PTPE. Because we expected difficulties in inserting the PTCD tube into the atrophic liver after PTPE, the PTCD tube was placed before PTPE. Previous reports showed that inserting the PTCD tube into the leaking bile duct was difficult $[20,21]$. To the best of our knowledge, no report was found of successful PTCD tube insertion into the atrophic liver after PTPE. The insertion of the PTCD tube into the bile duct of segment $\mathrm{V}$ was easier than the insertion into the bile duct of segment VIII, because the liver shape changed after hepatectomy and the bile duct of segment VIII was near the cavity of the bile leakage, making insertion technically difficult. In the past report, the retrograde transhepatic biliary drainage tube was inserted into the bile duct under open surgery, and bile duct ablation with ethanol was performed from the tube [20]. In this case, 6-Fr PTCD tube was successfully inserted to the normal size of the bile duct of segment $\mathrm{V}$ by an interventional radiologist before PTPE. PTPE decreased the liver capacity of segments V and VIII. Bile leakage was decreased from 150 to $50 \mathrm{~mL}$. Bile leakage was not cured by PTPE alone. Although bile duct ablation was performed with pure ethanol, this case report revealed that bile leakage was treated with PTPE because it was not cured by bile duct ablation alone [15].

TAE for treating bile leakage has been rarely reported, and the authors found only one case report [23]. However, it was reported that TAE can increase the risk of biliary infection, which may cause liver abscess after the procedure [14]. Therefore, TAE was not performed in our case.

\section{Conclusions}

We report a case of isolated bile leakage after partial hepatectomy that was successfully treated with combination therapy with PTPE and bile duct ablation with ethanol. It is important to diagnose the bile leakage type and to select the adequate treatment method.

\section{Abbreviations \\ CT: Computed tomography; DIC: Drip infusion cholangiography; FRLV: Functional remnant liver volume; MRI: Magnetic resonance imaging; POD: Postoperative day; PTCD: Percutaneous transhepatic cholangiography drainage; PTPE: Percutaneous transhepatic portal embolization}

\section{Authors' contributions}

$\mathrm{NH}, \mathrm{KA}, \mathrm{NI}, \mathrm{MT}, \mathrm{TI}, \mathrm{AW}, \mathrm{KS}, \mathrm{MM}$, and NK performed the surgery and perioperative management of the patient and helped in drafting th manuscript. KA, NI, MT, TI, AW, HK, and KS participated in revising the manuscript critically. All authors read and approved the final manuscript.

Ethics approval and consent to participate Not applicable.

Consent for publication

This patient consented to the reporting of this case in a scientific publication.

Competing interests

The authors declare that they have no competing interests.

\section{Publisher's Note}

Springer Nature remains neutral with regard to jurisdictional claims in published maps and institutional affiliations.

\section{Author details}

${ }^{1}$ Department of Hepatobiliary and Pancreatic Surgery, Gunma University, Graduate School of Medicine, 3-39-22 Showa-machi, Maebashi, Gunma 371-8511, Japan. Department of Diagnostic and interventional Radiology, Gunma University Hospital, 3-39-15 Showa-machi, Maebashi, Gunma 371-8511, Japan. ${ }^{3}$ Department of General Surgical Science, Gunma University, Graduate School of Medicine, Maebashi, Japan. 
Received: 22 April 2018 Accepted: 4 June 2018

Published online: 19 June 2018

\section{References}

1. Brooke-Smith M, Figueras J, Ullah S, Rees M, Vauthey JN, Hugh TJ, et al. Prospective evaluation of the International Study Group for Liver Surgery definition of bile leak after a liver resection and the role of routine operative drainage: an international multicentre study. HPB (Oxford). 2015;17:46-51.

2. Kyoden $Y$, Imamura H, Sano K, Beck Y, Sugawara Y, Kokudo N, et al. Value of prophylactic abdominal drainage in 1269 consecutive cases of elective liver resection. J Hepatobiliary Pancreat Sci. 2010;17:186-92.

3. Yang T, Zhang J, Lu JH, Yang GS, Wu MC, Yu WF. Risk factors influencing postoperative outcomes of major hepatic resection of hepatocellular carcinoma for patients with underlying liver diseases. World J Surg. 2011;35: 2073-82.

4. Cescon M, Vetrone G, Grazi GL, Ramacciato G, Ercolani G, Ravaioli M, et al. Trends in perioperative outcome after hepatic resection: analysis of 1500 consecutive unselected cases over 20 years. Ann Surg. 2009;249:995-1002.

5. Nakagawa K, Tanaka K, Nojiri K, Sawada Y, Kumamoto T, Ueda M, et al. Predictive factors for bile leakage after hepatectomy for hepatic tumors: a retrospective multicenter study with 631 cases at Yokohama Clinical Oncology Group (YCOG). J Hepatobiliary Pancreat Sci. 2017;24:33-41.

6. Capussotti L, Ferrero A, Vigano L, Sgotto E, Muratore A, Polastri R. Bile leakage and liver resection: where is the risk? Arch Surg. 2006;141:690-4. discussion 695

7. Erdogan D, Busch OR, van Delden OM, Rauws EA, Gouma DJ, van Gulik TM. Incidence and management of bile leakage after partial liver resection. Dig Surg. 2008;25:60-6.

8. Ishii H, Ochiai T, Murayama Y, Komatsu S, Shiozaki A, Kuriu Y, et al. Risk factors and management of postoperative bile leakage after hepatectomy without bilioenteric anastomosis. Dig Surg. 2011;28:198-204.

9. Nagano Y, Togo S, Tanaka K, Masui H, Endo I, Sekido H, et al. Risk factors and management of bile leakage after hepatic resection. World J Surg. 2003;27:695-8

10. Koch M, Garden OJ, Padbury R, Rahbari NN, Adam R, Capussotti L, et al. Bile leakage after hepatobiliary and pancreatic surgery: a definition and grading of severity by the International Study Group of Liver Surgery. Surgery. 2011; 149:680-8.

11. Itoh S, Yoshizumi T, Shirabe K, Kimura K, Okabe H, Harimoto N, et al. Functional remnant liver assessment predicts liver-related morbidity after hepatic resection in patients with hepatocellular carcinoma. Hepatol Res. 2017:47:398-404.

12. Fukuhisa H, Sakoda M, Hiwatashi K, lino S, Minami K, Hashiguchi M, et al. Surgical treatment for the excluded bile leakage from Spiegel lobe after right hemihepatectomy: a case report. Int J Surg Case Rep. 2017:39:159-63.

13. Sakamoto K, Tamesa T, Yukio T, Tokuhisa Y, Maeda Y, Oka M. Risk factors and managements of bile leakage after hepatectomy. World J Surg. 2016;40: 182-9.

14. Woo S, Chung JW, Hur S, Joo SM, Kim HC, Jae HJ, et al. Liver abscess after transarterial chemoembolization in patients with bilioenteric anastomosis: frequency and risk factors. AJR Am J Roentgenol. 2013;200:1370-7.

15. Sadakari Y, Miyoshi A, Ohtsuka T, Kohya N, Takahashi T, Matsumoto K, et al. Percutaneous transhepatic portal embolization for persistent bile leakage after hepatic resection: report of a case. Surg Today. 2008;38:668-71.

16. Tanaka S, Hirohashi K, Tanaka H, Shuto T, Lee SH, Kubo S, et al. Incidence and management of bile leakage after hepatic resection for malignant hepatic tumors. J Am Coll Surg. 2002;195:484-9.

17. Kyokane T, Nagino M, Sano T, Nimura Y. Ethanol ablation for segmental bile duct leakage after hepatobiliary resection. Surgery. 2002;131:111-3.

18. Matsumoto T, Iwaki K, Hagino Y, Kawano K, Kitano S, Tomonari K, et al. Ethanol injection therapy of an isolated bile duct associated with a biliarycutaneous fistula. J Gastroenterol Hepatol. 2002;17:807-10.

19. Sakaguchi T, Shibasaki Y, Morita Y, Oishi K, Suzuki A, Fukumoto K, et al. Postoperative bile leakage managed by interventional intrabiliary ethanol ablation. Hepatogastroenterology. 2011;58:157-60.

20. Shimizu T, Yoshida H, Mamada Y, Taniai N, Matsumoto S, Mizuguchi Y, et al. Postoperative bile leakage managed successfully by intrahepatic biliary ablation with ethanol. World J Gastroenterol. 2006:12:3450-2.

21. Kataoka M, Ooeda Y, Yoshioka S, Wakatsuki K, Tonooka T, Kawamoto J, et al. Percutaneous transhepatic ethanol ablation for postoperative bile leakage after a hepatectomy. Hepatogastroenterology. 2011;58:988-91.
22. Kyokane T, Nagino M, Oda K, Nimura Y. An experimental study of selective intrahepatic biliary ablation with ethanol. J Surg Res. 2001;96:188-96.

23. Ikeda A, Noda T, Hatano H, Takata A, Hirota M, Oshima K, et al. A case of intractable bile leakage after surgery for gallbladder cancer successfully treated by transcatheter arterial embolization. Gan To Kagaku Ryoho. 2015; 42:1734-6.

\section{Submit your manuscript to a SpringerOpen ${ }^{\circ}$ journal and benefit from:}

- Convenient online submission

- Rigorous peer review

- Open access: articles freely available online

- High visibility within the field

- Retaining the copyright to your article

Submit your next manuscript at $>$ springeropen.com 